\title{
Construction and analysis of a spinal cord injury competitive endogenous RNA network based on the expression data of long noncoding, micro- and messenger RNAs
}

\author{
LINBANG WANG $^{1}$, BING WANG ${ }^{2}$, JINGKUN LIU $^{3}$ and ZHENGXUE QUAN ${ }^{1}$ \\ ${ }^{1}$ Department of Orthopedic Surgery, The First Affiliated Hospital of Chongqing Medical University, Chongqing 400016; \\ ${ }^{2}$ Laboratory of Environmental Monitoring, Shaanxi Province Health Inspection Institution, Xi'an, Shaanxi 710077; \\ ${ }^{3}$ Department of Orthopedics, Honghui Hospital, Xi'an Jiaotong University, Xi'an, Shaanxi 710054, P.R. China
}

Received May 21, 2018; Accepted February 1, 2019

DOI: $10.3892 / \mathrm{mmr} .2019 .9979$

\begin{abstract}
Spinal cord injury (SCI) results from trauma and predominantly affects the young male population. SCI imposes major and permanent life changes, and is associated with high future mortality and disability rates. Long non-coding RNAs (lncRNAs) have recently been demonstrated to serve critical roles in a broad range of biological processes and to be expressed in various diseases, including in SCI. However, the precise mechanisms underlying the roles of lncRNAs in SCI pathogenesis remain unexplored. In the present study, the aim was to identify critical differentially expressed lncRNAs in SCI based on the competing endogenous RNA (ceRNA) hypothesis by mining data from the Gene Expression Omnibus database of the National Center for Biotechnology Information and to unveil the functions of these lncRNAs. Different approaches and tools were employed to establish a network consisting of 13 lncRNA, 93 messenger RNA and 9 microRNA nodes, with a total of 202 edges. Three node lncRNAs were identified based on the degree distribution of the nodes, and their corresponding subnetworks were subsequently constructed. Based on these subnetworks, the biological pathways and interactions of these 3 lncRNAs were detailed using FunRich software
\end{abstract}

Correspondence to: Professor Zhengxue Quan, Department of Orthopedic Surgery, The First Affiliated Hospital of Chongqing Medical University, 1 Youyi Road, Yuanjiagang, Yuzhong, Chongqing 400016, P.R. China

E-mail: quanzx18@126.com

Abbreviations: SCI, spinal cord injury; lncRNAs, long non-coding RNAs; ceRNA, competing endogenous RNA; mRNAs, messenger RNAs; miRNAs, microRNAs; GEO, Gene Expression Omnibus; NCBI, National Center for Biotechnology Information; AP-1, activator protein 1; CDC42, cell division cycle 42; S1P1, sphingosine-1-phosphate receptor 1; Arf6, ADP-ribosylation factor 6; PI3K, phosphoinositide 3-kinase; Akt, protein kinase B; mTOR, mammalian target of rapamycin; mTORC1, mTOR complex 1

Key words: SCI, autophagy, ceRNA, lncRNA, PI3K/AKT/mTOR (version 3.0). The primary results of the 3 lncRNA enrichment analyses were that they were associated with autophagy, extracellular communication and transcription factor networks, respectively. The phosphoinositide 3-kinase/protein kinase $\mathrm{B} / \mathrm{mammalian}$ target of rapamycin signaling pathway of XR_350851 was the classic autophagy pathway, indicating that XR_350851 may regulate autophagy in SCI. The possible role of XR_350851 in SCI revealed in the current study based on the regulatory mechanism of ceRNAs has uncovered a new repertoire of molecular factors with potential as novel biomarkers and therapeutic targets in SCI.

\section{Introduction}

Spinal cord injury (SCI) is a serious and disabling disease associated with a range of symptoms, including severe movement dysfunction, muscle weakness, and sensory changes (1). Based on pathological patterns, SCI can be classified as a primary or secondary injury. Although little is known regarding the pathocellular mechanisms of SCI, secondary damage following primary SCI are considered to involvea wide range of pathologies, including neural inflammation, demyelination, axonal degeneration, and oligodendrocyte and neuronal cell death $(2,3)$. Great efforts have been made to improve the functional outcomes of patients with SCI; however, therapeutic advances have thus far been limited (4). Therefore, the specific objective of the present study was to identify biomarkers and therapeutic targets for SCI.

A large number of studies have implicated apoptosis, autophagy, inflammation and endoplasmic reticulum stress as important features of SCI; however, regulatory mechanisms based on crosstalk between these factors remain to be delineated (5). A strong association between autophagy and acute neurodegeneration caused by SCI has previously been reported in the literature (6). Autophagy is a highly conserved evolutionary phenomenon of intracellular degradation that maintains the homeostasis of cells by inducing rapid self-clearance or by degrading supramolecular structures $(7,8)$. Similar to cell division, differentiation and death, autophagy dysfunctionis associated with numerous diseases, including cancer, and cardiovascular and neurodegenerative diseases $(9,10)$. 
Pott et al (11) demonstrated that inadequate autophagy in intestinal epithelial cells increases the induction of apoptosis and potentially impairs barrier integrity due to inflammatory stimuli. A study conducted by Papadakis et al (12) also indicated that autophagy may have a protective role in an oxygenglucose-deprivation neuronal and cerebral ischemia model. However, recent evidence (10) suggests that whether autophagy is protective or detrimental may be based on the activation status of the cell, as well as other factors. Notably, increased or decreased autophagy potentially contributes to a variety of diseases and pathological conditions (13).

Among the RNAs of different molecular ranges, the sequences of long non-coding RNAs (lncRNAs) are the least evolutionarily conserved. At the cellular level, epigenetic modulation is one of the salient roles of lncRNAs, although lncRNAs can also regulate gene expression through transcription, alternative splicing, RNA translation and organization of important structures for RNA processing. An abundance of chromatin modification complexes can be targeted by lncRNAs to reconstruct the structure and/or expression of their adjacent genes (14). Prior studies have noted the critical roles of lncRNAs in the pathogenesis of various neurological diseases, including SCI. For instance, there is evidence that the IncRNA-XIST significantly aids in the recovery from SCI by inhibiting apoptosis (15). Qiao et al (16) also suggested that the lncRNA MALAT1 has a neuroprotective role in spinal cord ischemic/reperfusion injury, where it acts by regulating miR-204. Furthermore, Zhou et al (17) confirmed that MALAT1 can inhibit acute SCI by inhibiting the inflammatory response of microglial cells.

Recent evidence has indicated that IncRNAs can regulate the expression of messenger RNAs (mRNAs) by competitively binding to microRNAs (miRNAs) and acting ascompeting endogenous RNAs (ceRNAs), which can systematically functionalize non-coding transcripts based on competitive sharing of miRNAs (18). It is considered that lncRNA transcripts function as competing endogenous RNAs (ceRNAs) or natural microRNA sponges that contain numerous miRNA binding sites. Salmena et al (19) proposed the 'ceRNA hypothesis', according to which the expression of a specific miRNA can be temporarily reduced due to ceRNA. In addition, based on the ceRNA hypothesis, scholars discovered a large-scale regulatory network in the transcriptome based on miRNA binding sites, which greatly expands the information availableon human functional genetics and details a network that may serve a critical role in cancer pathology $(19,20)$.

In the present study, a global triple network was generated based on the ceRNA hypothesis by mining data from the Gene Expression Omnibus (GEO), which is curated by the National Center for Biotechnology Information (NCBI). Based on the resulting network, target lncRNAs associated with SCI were identified. Furthermore, a subnetwork of node lncRNAs was obtained from the ceRNA network, which facilitated enrichment and identification of lncRNA pathways and functions. The flow chart for target lncRNA selection is presented in Fig. 1.

\section{Materials and methods}

Data sources. GEO (www.ncbi.nlm.nih.gov/geo) is currently the largest fully public gene expression resource data repository, from which thousands of experiments and tens of millions of gene expression profiles can be queried and downloaded. Data examined in the present study were obtained from the NCBI GEO database. The raw mRNA expression data were downloaded from the series GSE464 (www.ncbi.nlm.nih. gov/geo/query/acc.cgi?acc=GSE464) and GSE5296 (www.ncbi. nlm.nih.gov/geo/query/acc.cgi?acc=GSE5296). The lncRNA data were obtained from the aforementioned datasets by repurposing the probes in the RG_U34A, RG_U34B, RG_U34C and Mouse430_2 arrays of the Affymetrix annotation platform (www.thermofisher.com/cn/en/home/life-science/microarrayanalysis/microarray-data-analysis/genechip-array-annotationfiles.html) (21). Data on miRNA were obtained from GEO series GSE19890 (www.ncbi.nlm.nih.gov/geo/query/acc. cgi?acc $=$ GSE19890).

Raw data analysis. Subsequent tologarithmic processing, the two mRNA expression datasets were merged using Perl script (www.perl.org/), and batch effects were corrected using the Combat method from the sva and limma packages (22-24). Differential mRNA expression analysis between SCI and normal samples was performed using the limma package (bioconductor. org/packages/release/bioc/html/limma.html) (25). mRNAs with a P-value of $<0.05$ and $\log _{2}$ fold-change $\left(\log _{2} \mathrm{FC}\right)$ of $>1.5$ were considered to be differentially expressed. Differential lncRNA and miRNA expression analysis between SCI and normal samples was performed using the Morpheus platform (https://software.broadinstitute.org/morpheus/) based on the significance analysis of microarrays method, and the threshold was also set tolog $\log _{2} \mathrm{FC}>1.5$ and $\mathrm{P}<0.05$.

Screening and pairwise matching of lncRNAs, miRNAs and mRNAs. Pairwise matching of differentially expressed lncRNAs, miRNAs and mRNAs was performed using RNAhybrid (bibiserv.cebitec.uni-bielefeld.de/rnahybrid), LncTar (www.cuilab.cn/lnctar), TargetScan Human version 7.1 (www.targetscan.org/vert_71/) and DIANA Tools (diana. imis.athena-innovation.gr/DianaTools/index.php? $r=$ microT_ CDS/index). The miRNA binding sites on lncRNAs were predicted using RNA hybrid (26), which is a tool used to calculate the minimum free energy hybridization of a long and short RNA. LncTar (27) is a software used for predicting putative interactions between mRNAs and lncRNAs based on free energy minimization. In addition, TargetScan (28) and DIANA $(29,30)$ were synchronously used to predict differentially expressed pairs of miRNAs and mRNAs. TargetScan predicts the biological targets of miRNAs by searching for the presence of conserved $8 \mathrm{mer}, 7 \mathrm{mer}$ and 6 mer sites by matching the seed region of each miRNA (31). In mammals, predictions were ranked based on the predicted efficacy of targeting as calculated using the cumulative weighted context++ scores of the sites. In the current study, the predicted mRNAs were set to a cumulative weighted context ++ score of $\leq-0.4$ in TargetScan (28). The arsenal of DIANA Tools included the target prediction algorithms of microT v4 and microT-CDS, where microT-CDS was used to predict the miRNA and mRNA pairs in the present study. In order to avoid data omission, the two online websites (TargetScan and DIANA) were used for prediction, and their results were combined. 


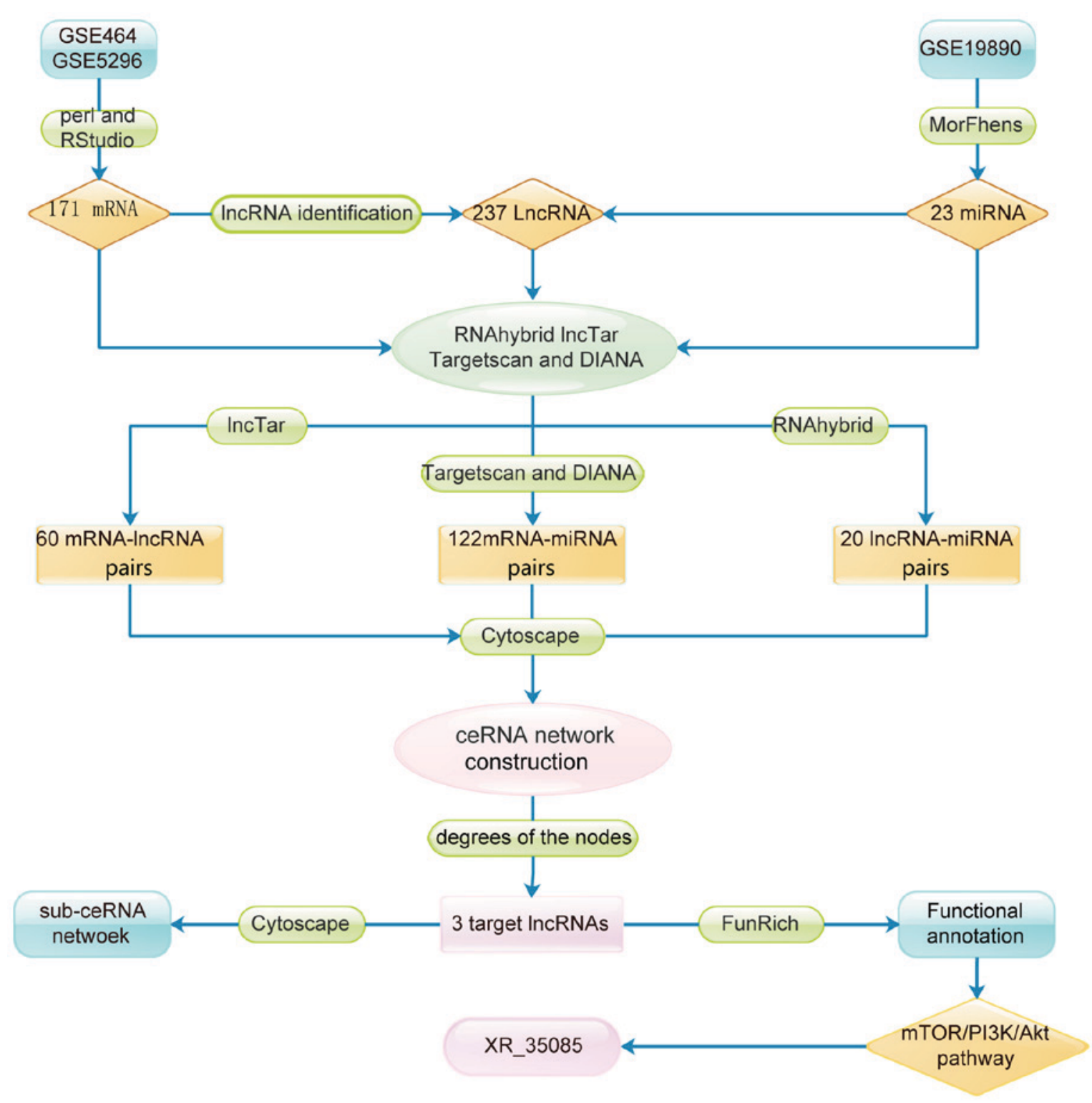

Figure 1. Flow chart for the target lncRNA selection.

Construction of the ceRNA network. For a given lncRNA-mRNA pair, which included negatively co-expressed mRNAs and lncRNAstargeted with a specified common miRNA, co-expression competing triplets were identified based on the ceRNA theory (32). Using existing miRNA target online software (RNAhybrid, LncTar, TargetScan and DIANA Tools), lncRNA-miRNA, lncRNA-mRNA and miRNA-mRNA interactions were confirmed. Using an in-house Perl script, the ceRNA associations were integrated (33). To elucidate the roles of lncRNAs, miRNAs and mRNAs within the regulatory ceRNA network, their interactive and visual mediated network was then created using Cytoscape software, version 3.5.0 (34).

Molecular function analysis of the ceRNA network. To elucidate the molecular functions within the ceRNA network, mRNAs in the network were analyzed using FunRich software (version 3.0; www.funrich.org/). The FunRich tool allows users to assign the biological process, cellular component and molecular function terms, as well as biological pathways, protein domains, sites of expression, clinical phenotypes and transcription factors, to enriched and depleted factors (35).

Reconstruction of a sub-ceRNA network using node lncRNAs. Based on the established ceRNA network, network analysis was subsequently performed to investigate the degree distribution of the nodes (36). Three node IncRNAs were screened based on the degrees of the nodes, while miRNA and mRNA pairing with the lncRNAs was also examined. Accordingly, three sub-ceRNA networks were established with the Cytoscape software (version 3.5.0).

Functional annotation enrichment analysis. Biological pathway functional enrichment analyses were conducted using FunRich software, version 3.0. This software was used to visualize and assess pathway interactions of the lncRNA-targeted mRNAs. This tool also highlighted nodes that were enriched in specific pathways and allowed for the creation of subnetworks based on these highlighted nodes (35). 


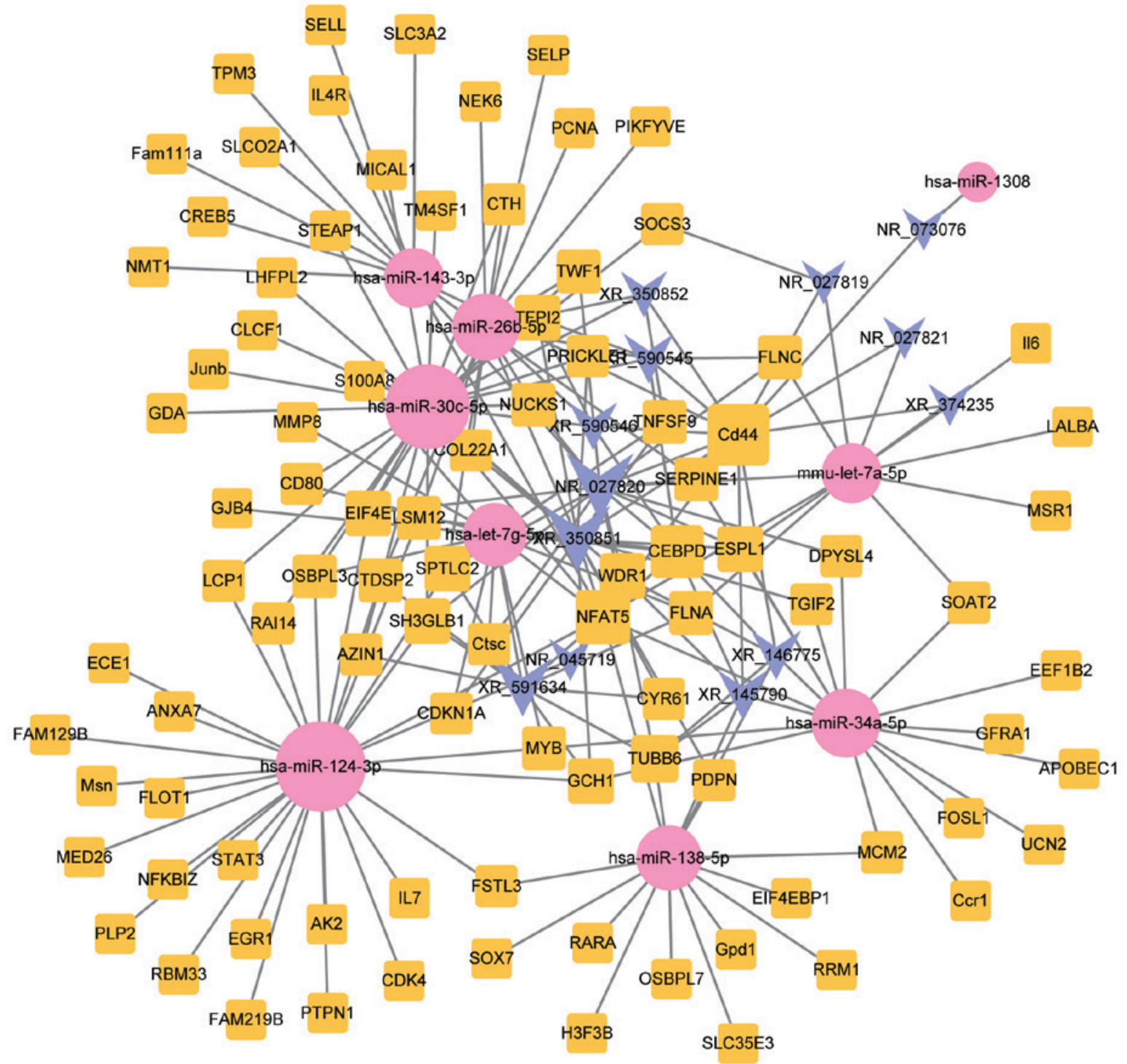

Figure 2. Competing endogenous RNA network of the lncRNA-miRNA-mRNA. The network is comprised of $13 \operatorname{lncRNAs,} 9$ miRNAs and 93 mRNAs, with a total of 202 edges. Purple inverted triangles indicate the lncRNAs, pink circles indicate the miRNAs, and orange squares indicate the mRNAs, these sizes are positively correlated with the degrees of the nodes. IncRNA, long noncoding RNA; mRNA, messenger RNA; miRNA, microRNA.

\section{Results}

Screening of differentially expressed lncRNAs, miRNAs and $m R N A s$. Following logarithmic processing and batch correction, the microarray data were analyzed by Morpheus and limma packages. In total, 171 mRNAs and 237 lncRNAs that were overexpressed in SCI were identified according to the threshold of $\log _{2} \mathrm{FC}>1.5$ and $\mathrm{P}<0.05$. In addition, 23 miRNAs that were downregulated in SCI were selected using Morpheus software (Fig. 1).

Pairwise matching of lncRNAs, miRNAs and mRNAs. Using RNAhybrid and LncTar, 20 mature miRNA-lncRNA pairs and 60 lncRNA-mRNA pairs were respectively predicted. Furthermore, a total of 122 unique mature miRNA-mRNA pairs, predicted by both TargetScan and DIANA, were selected as reliable interaction pairs.

Construction of the IncRNA-miRNA-mRNA ceRNA network. In order to elucidate the interactions between lncRNAs, miRNAs and mRNAs in SCI, a ceRNA network was established. As shown in Fig. 2, this network consisted of 13 lncRNA, 93 mRNA and 9 miRNA nodes, with a total of 202 edges.

Molecular annotation of IncRNAs based on the ceRNA network. To identify possible mechanisms associated with SCI, 75 molecular function modules were selected using FunRich 3.0 software. As displayed in Table I, the primary modules included calcium ion-binding, cysteine-type peptidase activity, chemokine activity, auxiliary transport protein activity, cytokine activity, hormone binding, steroid binding, protein-tyrosine kinase activity, transcription factor activity and cytoskeletal anchoring activity. The top 10 critical molecular functions of mRNAs in the ceRNA network are presented in Fig. 3. The percentage and P-values of critical molecular functions are also presented; there was a higher proportion of transcription factor activity (7.6\%), auxiliary transport protein activity $(4 \%)$ and calcium ion-binding (3.6\%; Fig. 3). 
Table I. A total of 75 molecular function modules potentially involved in spinal cord injury.

\begin{tabular}{|c|c|c|}
\hline Molecular function & Fold enrichment & P-value \\
\hline Calcium ion binding & 3.760905 & 0.000706 \\
\hline Cysteine-type peptidase activity & 6.730617 & 0.002923 \\
\hline Chemokine activity & 5.841835 & 0.004888 \\
\hline Auxiliary transport protein activity & 2.321344 & 0.011811 \\
\hline Cytokine activity & 3.615564 & 0.012769 \\
\hline Hormone binding & 77.225850 & 0.012949 \\
\hline Steroid binding & 77.225850 & 0.012949 \\
\hline Protein-tyrosine kinase activity & 6.115491 & 0.012960 \\
\hline Transcription factor activity & 1.743523 & 0.013665 \\
\hline Cytoskeletal anchoring activity & 5.958724 & 0.013908 \\
\hline Receptor activity & 2.141300 & 0.019646 \\
\hline Acyltransferase activity & 3.870462 & 0.020167 \\
\hline Ubiquitin-like-protein-specific protease activity & 38.805030 & 0.025730 \\
\hline Translation regulator activity & 3.065792 & 0.042320 \\
\hline Metallopeptidase activity & 3.065792 & 0.042320 \\
\hline Complement activity & 5.541734 & 0.050627 \\
\hline Ion channel activity & 3.521433 & 0.054121 \\
\hline Intracellular ligand-gated ion channel activity & 5.172408 & 0.057295 \\
\hline Ligand-dependent nuclear receptor activity & 4.433704 & 0.075174 \\
\hline Lipid kinase activity & 4.433704 & 0.075174 \\
\hline Phosphoprotein phosphatase activity & 12.978060 & 0.075229 \\
\hline Guanylate cyclase activity & 9.737592 & 0.099030 \\
\hline Lipid transporter activity & 8.656838 & 0.110701 \\
\hline Transcription factor binding & 7.792019 & 0.122222 \\
\hline Hydrolase activity & 1.905825 & 0.124300 \\
\hline Lipid binding & 7.084297 & 0.133594 \\
\hline Serine-type peptidase activity & 2.371695 & 0.134282 \\
\hline RNA methyltransferase activity & 6.494431 & 0.144819 \\
\hline Transmembrane receptor activity & 2.984502 & 0.145688 \\
\hline Cytoskeletal protein binding & 1.774696 & 0.153605 \\
\hline Oxidoreductase activity & 1.923332 & 0.156614 \\
\hline Water channel activity & 5.567317 & 0.166836 \\
\hline Kinase activity & 5.567317 & 0.166836 \\
\hline Receptor signaling protein tyrosine phosphatase activity & 4.871837 & 0.188289 \\
\hline Protein binding & 1.701421 & 0.210739 \\
\hline Deaminase activity & 4.103004 & 0.219441 \\
\hline Carboxy-lyase activity & 3.897957 & 0.229558 \\
\hline Phosphorylase activity & 3.897957 & 0.229558 \\
\hline Peroxidase activity & 3.897957 & 0.229558 \\
\hline Transaminase activity & 3.389748 & 0.259133 \\
\hline Kinase regulator activity & 3.389748 & 0.259133 \\
\hline Hormone activity & 3.118677 & 0.278218 \\
\hline Helicase activity & 3.118677 & 0.278218 \\
\hline Protein tyrosine phosphatase activity & 3.118677 & 0.278218 \\
\hline Transcription regulator activity & 1.207568 & 0.282903 \\
\hline Heat shock protein activity & 2.887749 & 0.296813 \\
\hline Protease inhibitor activity & 1.651143 & 0.344255 \\
\hline Extracellular matrix structural constituent & 1.400216 & 0.364123 \\
\hline Galactosyltransferase activity & 2.052042 & 0.390878 \\
\hline Extracellular ligand-gated ion channel activity & 1.901929 & 0.414282 \\
\hline Ribonucleoprotein & 1.901929 & 0.414282 \\
\hline Growth factor activity & 1.410999 & 0.417894 \\
\hline
\end{tabular}


Table I. Continued.

\begin{tabular}{|c|c|c|}
\hline Molecular function & Fold enrichment & P-value \\
\hline Ligase activity & 1.385805 & 0.426817 \\
\hline Isomerase activity & 1.732906 & 0.444099 \\
\hline Phospholipase activity & 1.732906 & 0.444099 \\
\hline Transporter activity & 1.073903 & 0.470688 \\
\hline DNA binding & 1.063906 & 0.474045 \\
\hline Chaperone activity & 1.231838 & 0.487156 \\
\hline Cell adhesion molecule activity & 1.086772 & 0.490374 \\
\hline Transmembrane receptor protein tyrosine kinase activity & 1.392575 & 0.518527 \\
\hline Peptidase activity & 1.344563 & 0.530951 \\
\hline Structural constituent of cytoskeleton & 1.132939 & 0.531709 \\
\hline Defense/immunity protein activity & 1.278448 & 0.548991 \\
\hline Motor activity & 0.987193 & 0.643607 \\
\hline RNA binding & 0.846085 & 0.700779 \\
\hline Receptor signaling complex scaffold activity & 0.721871 & 0.790031 \\
\hline Receptor binding & 0.604590 & 0.814912 \\
\hline Voltage-gated ion channel activity & 0.599939 & 0.817325 \\
\hline Transferase activity & 0.499956 & 0.870160 \\
\hline Protein serine/threonine kinase activity & 0.515677 & 0.903888 \\
\hline Catalytic activity & 0.582086 & 0.916616 \\
\hline Ubiquitin-specific protease activity & 0.411724 & 0.957707 \\
\hline Structural molecule activity & 0.289945 & 0.970716 \\
\hline G-protein coupled receptor activity & 0.105974 & 0.999944 \\
\hline Molecular function unknown & 0.557085 & 0.999999 \\
\hline
\end{tabular}

Target lncRNA-miRNA-mRNA subnetwork. To illuminate the competitive mechanisms and potential biological functions of lncRNAs in SCI, the degree distribution of nodes in the ceRNA network were investigated using Cytoscape software. The horizontal axis of Fig. 4 represents the degree of RNA in the ceRNA network. As presented in Fig. 4 and Table II, hsa-miR-124-3p had the highest degree of node, with a node value of 29. In Fig. 5, the subnetworks of XR_350851, NR_027820, and XR_591634, respectively, are presented, showing how the mRNA directly or indirectly is associated with the lncRNA.

Enrichment analysis of lncRNA-targeted mRNAs. Biological pathway annotation analysis of significantly differentially expressed mRNAs in the ceRNA subnetwork revealed relevant pathways and molecular interactions with associated genes. Based on FunRich software enrichment analyses, 263, 139 and 174 biological pathways were identified to be the components associate with the genes in the XR_350851, NR_027820 and XR_591634 subnetworks, respectively. As shown in Fig. 6, the critical biological pathways in the XR_350851 subnetwork included the activator protein 1 (AP-1) transcription factor network, integrin-linked kinase signaling, cell division cycle 42 (CDC42) signaling events, sphingosine-1-phosphate receptor 1 (S1P1) pathway, ADP-ribosylation factor 6 (Arf6) trafficking events, epidermal growth factor receptor 1 downstream signaling, class I phosphoinositide 3-kinase (PI3K) signaling events mediated by protein kinase $\mathrm{B}(\mathrm{Akt})$, insulin pathway,
Table II. Differentially expressed genes of the competing endogenous RNAs (node degree $\geq 5$ ).

\begin{tabular}{llc}
\hline Number & \multicolumn{1}{c}{ RNA name } & Degree \\
\hline 1 & hsa-miR-124-3p & 29 \\
2 & hsa-miR-30c-5p & 26 \\
3 & hsa-miR-34a-5p & 17 \\
4 & NR_027820 & 16 \\
5 & hsa-miR-26b-5p & 16 \\
6 & XR_350851 & 15 \\
7 & hsa-miR-138-5p & 15 \\
8 & hsa-let-7g-5p & 14 \\
9 & Cd44 & 13 \\
10 & hsa-miR-143-3p & 12 \\
11 & mmu-let-7a-5p & 12 \\
12 & XR_591634 & 11 \\
13 & NFAT5 & 9 \\
14 & CEBPD & 8 \\
15 & XR_590545 & 6 \\
16 & XR_145790 & 6 \\
17 & XR_146775 & 6 \\
18 & XR_590546 & 5 \\
19 & TUBB6 & 5 \\
\hline & &
\end{tabular}




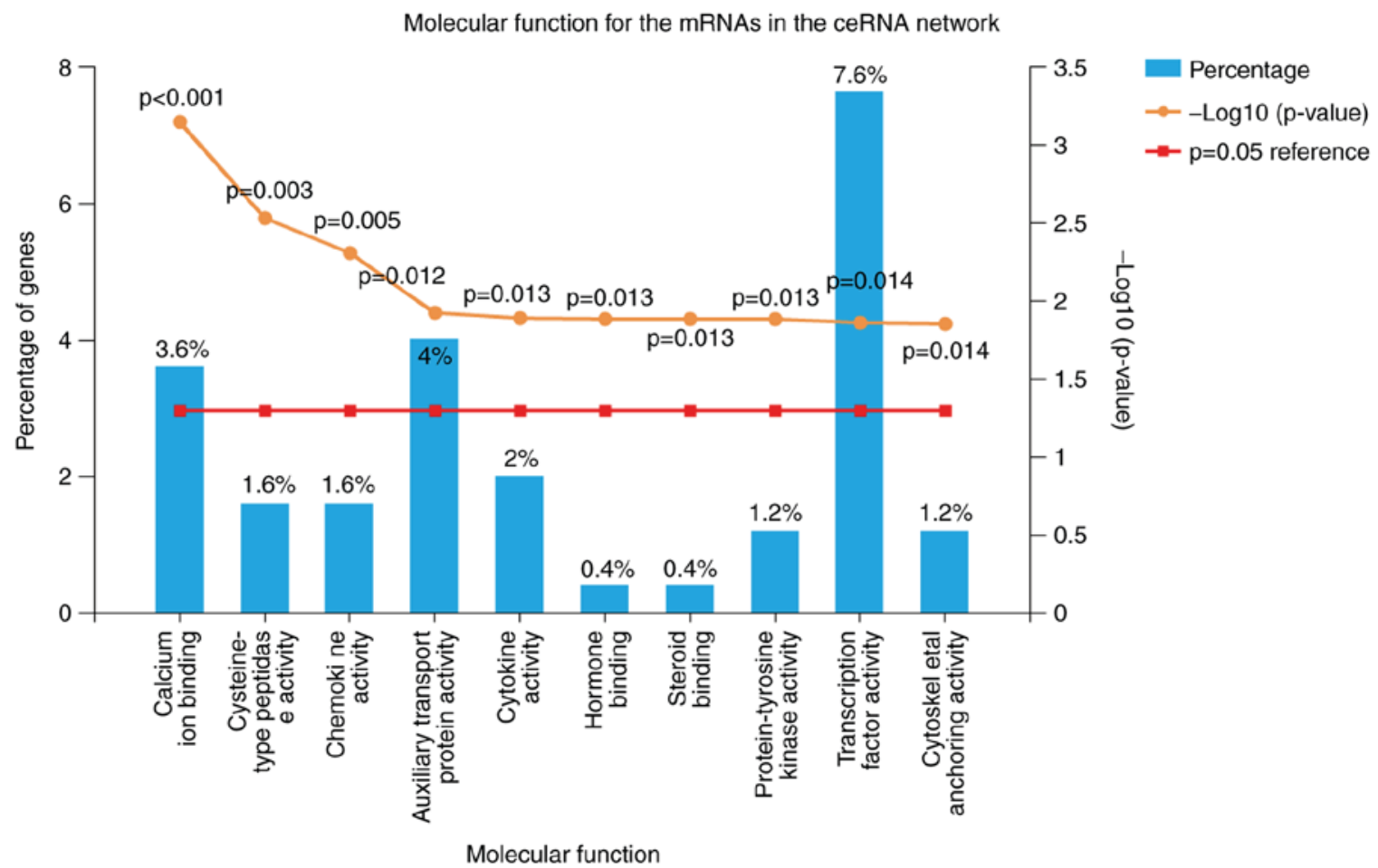

Figure 3. Top 10 critical molecular functions of the mRNAs in the ceRNA network. mRNA, messenger RNA; ceRNA, competing endogenous RNA.

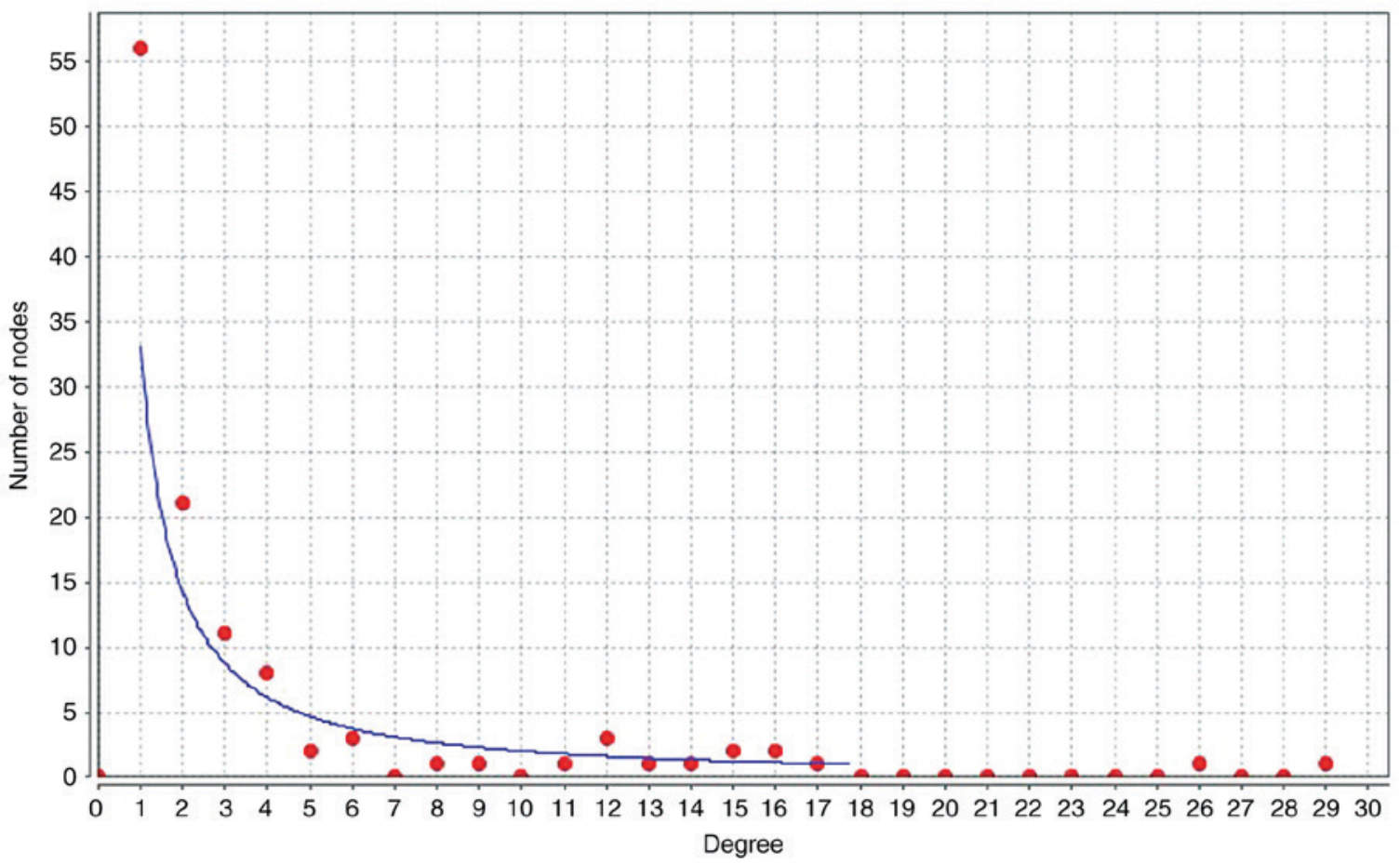

Figure 4. Degree distribution of nodes in the competing endogenous RNA network.

mammalian target of rapamycin (mTOR) signaling pathway and Arf6 downstream pathway. In addition, it was observed that the significant biological pathways identified for the subnetwork of NR_027820 were the following: Cell-extracellular matrix interactions, platelet degranulation, response to elevated platelet cytosolic $\mathrm{Ca}^{2+}$, cell junction organization, ceramide biosynthesis, hemostasis, cell-cell communication, release of eukaryotic translation initiation factor 4E, platelet activation, signaling and aggregation, and glycoprotein Ib-IX-V activation signaling (Fig. 7). Furthermore, the top 10 biological pathways for the XR_591634 subnetwork were as follows: C-MYB transcription factor network, AP-1 transcription factor network, integrin-linked kinase signaling, CDC42 signaling events, non-canonical Wnt signaling pathway, regulation of 

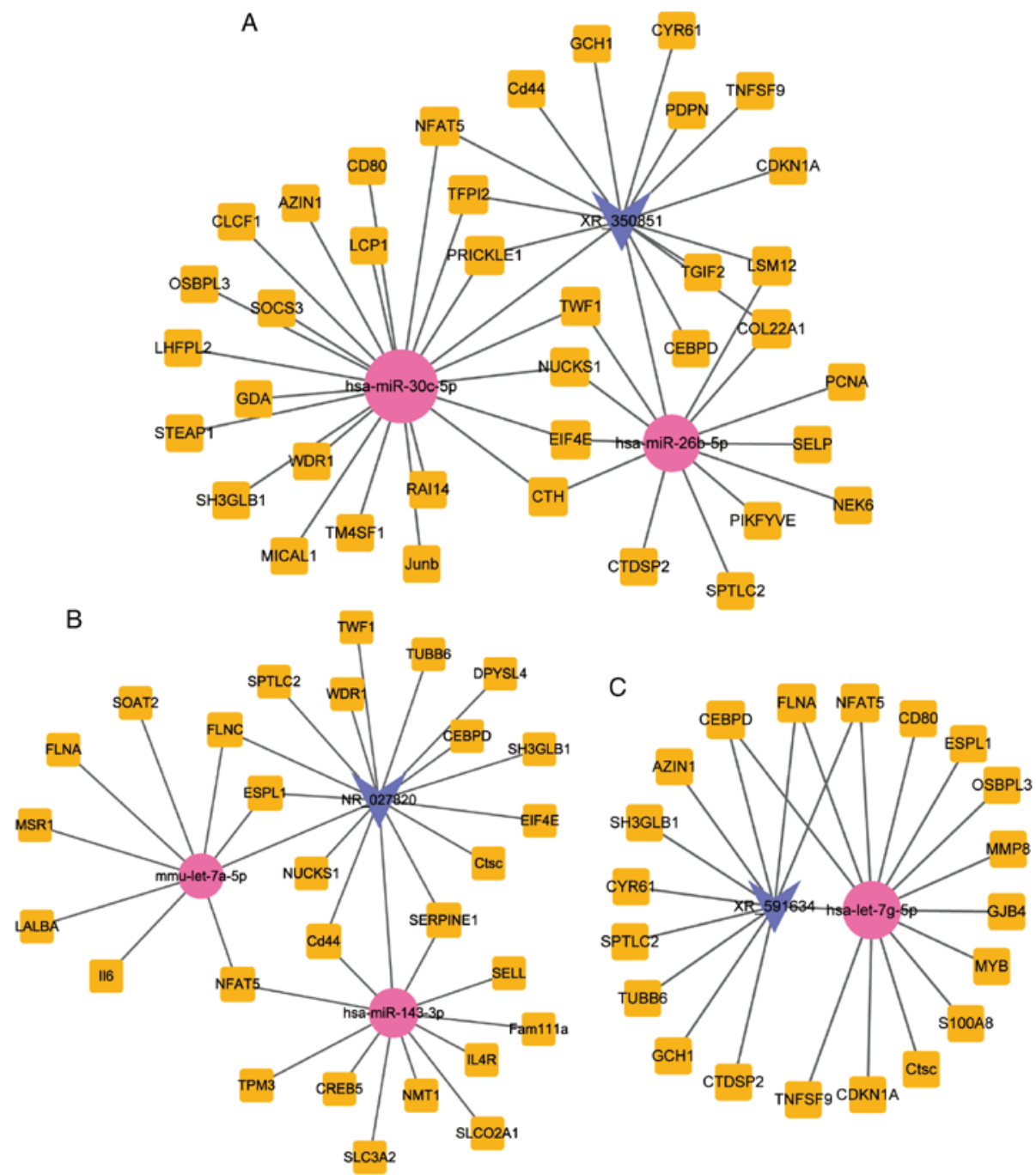

Figure 5. Sub-ceRNA networks of three node lncRNAs, including (A) XR_350851, (B) NR_027820 and (C) XR_591634 are displayed. Purple inverted triangles indicate the lncRNAs, pink circles indicate the miRNAs, and orange squares indicate the mRNAs, while the sizes of these shapes are positively correlated with the degrees of the nodes. ceRNA, competing endogenous RNA; IncRNA, long noncoding RNA; mRNA, messenger RNA; miRNA, microRNA.

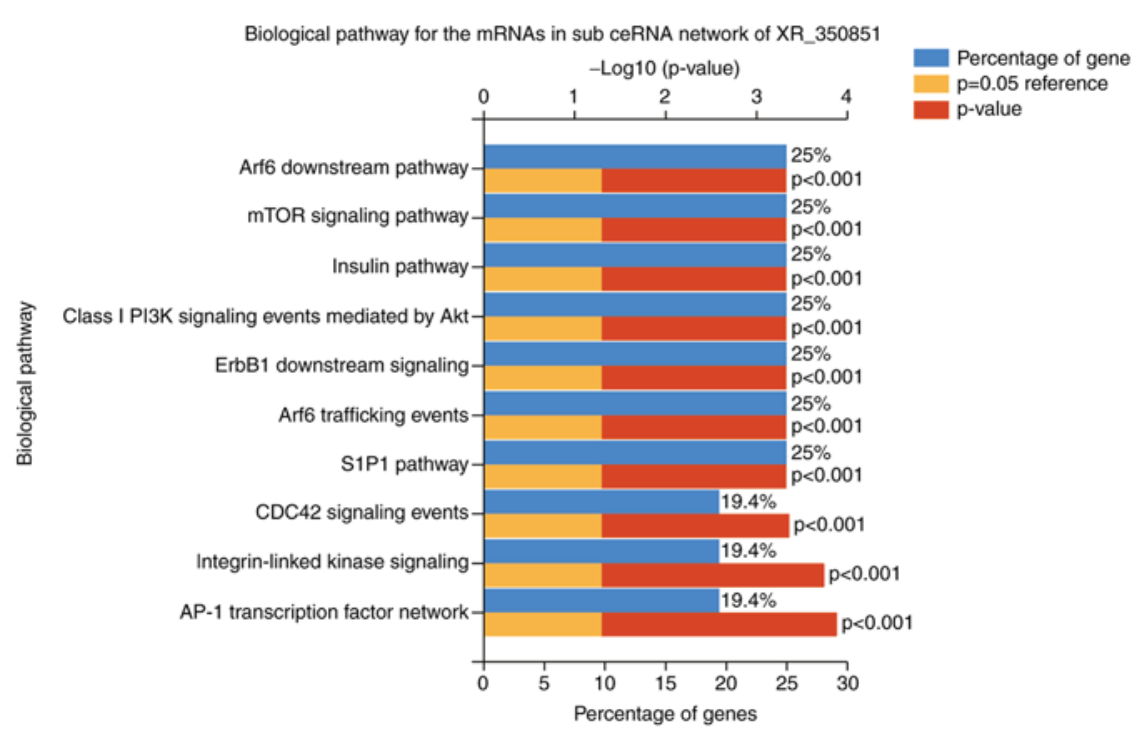

Figure 6. Top 10 biological pathways of the mRNAs in the sub-ceRNA network of the long noncoding RNA XR_350851. The top identified pathways included the AP-1 transcription factor network, integrin-linked kinase signaling, CDC42 signaling events, S1P1 pathway, Arf6 trafficking events, ErbB1 downstream signaling, class I PI3K signaling events mediated by Akt, insulin pathway, mTOR signaling pathway and Arf6 downstream pathway. mRNA, messenger RNA; ceRNA, competing endogenous RNA; AP-1, activator protein 1; CDC42, cell division cycle 42; S1P1, sphingosine-1-phosphate receptor 1; Arf6, ADP-ribosylation factor 6; ErbB1, epidermal growth factor receptor 1; mTOR, mammalian target of rapamycin; PI3K, phosphoinositide 3-kinase; Akt, protein kinase B. 


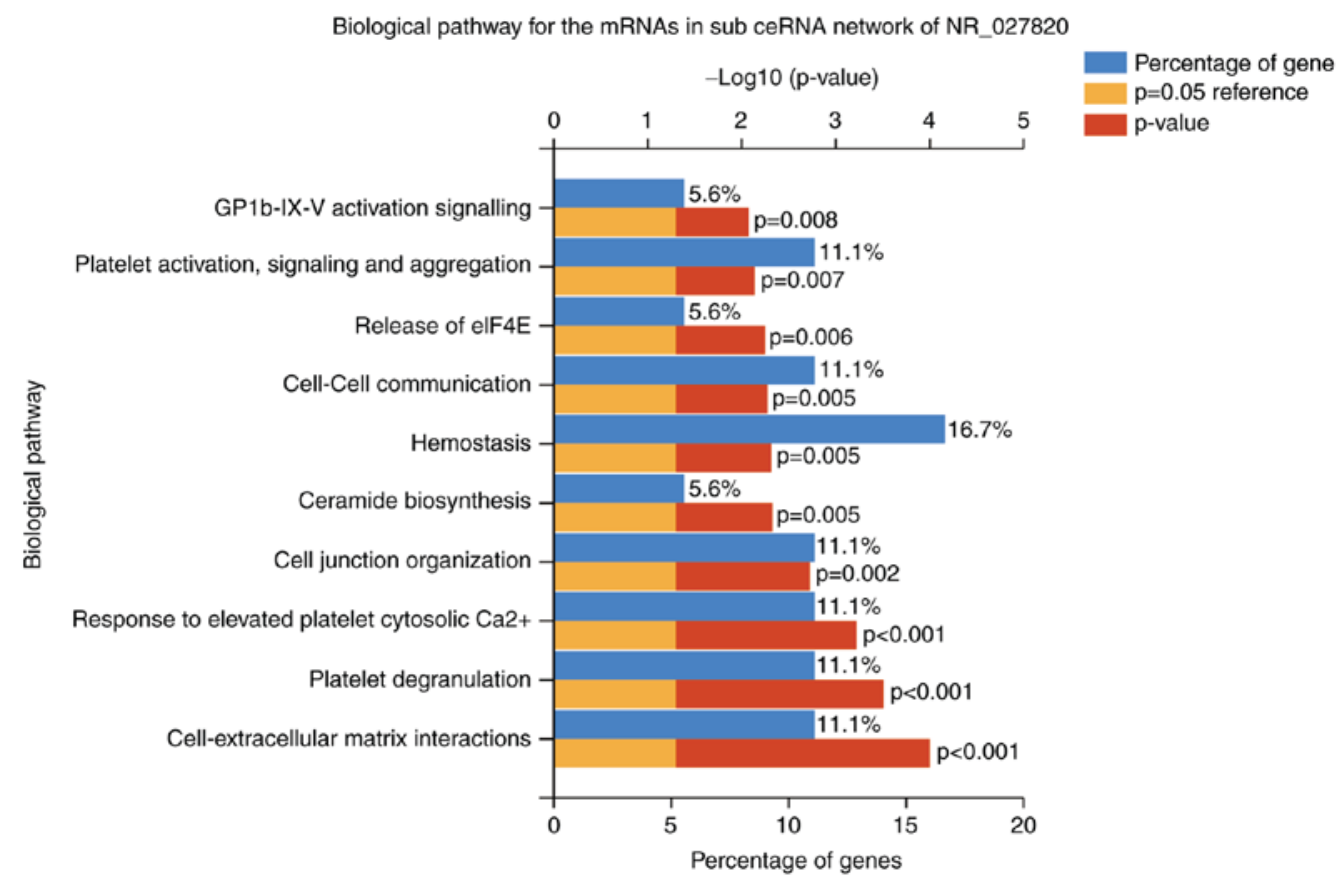

Figure 7. Top 10 biological pathways of the mRNAs in the sub-ceRNA network of the long noncoding RNA NR_027820. These pathways included the cell-extracellular matrix interactions, platelet degranulation, response to elevated platelet cytosolic $\mathrm{Ca}^{2+}$, cell junction organization, ceramide biosynthesis, hemostasis, cell-cell communication, release of eIF4E, platelet activation, signaling and aggregation, GPIb-IX-V activation signaling. mRNA, messenger RNA; ceRNA, competing endogenous RNA; eIF4E, eukaryotic translation initiation factor 4E; GPIb-IX-V, glycoprotein Ib-IX-V.

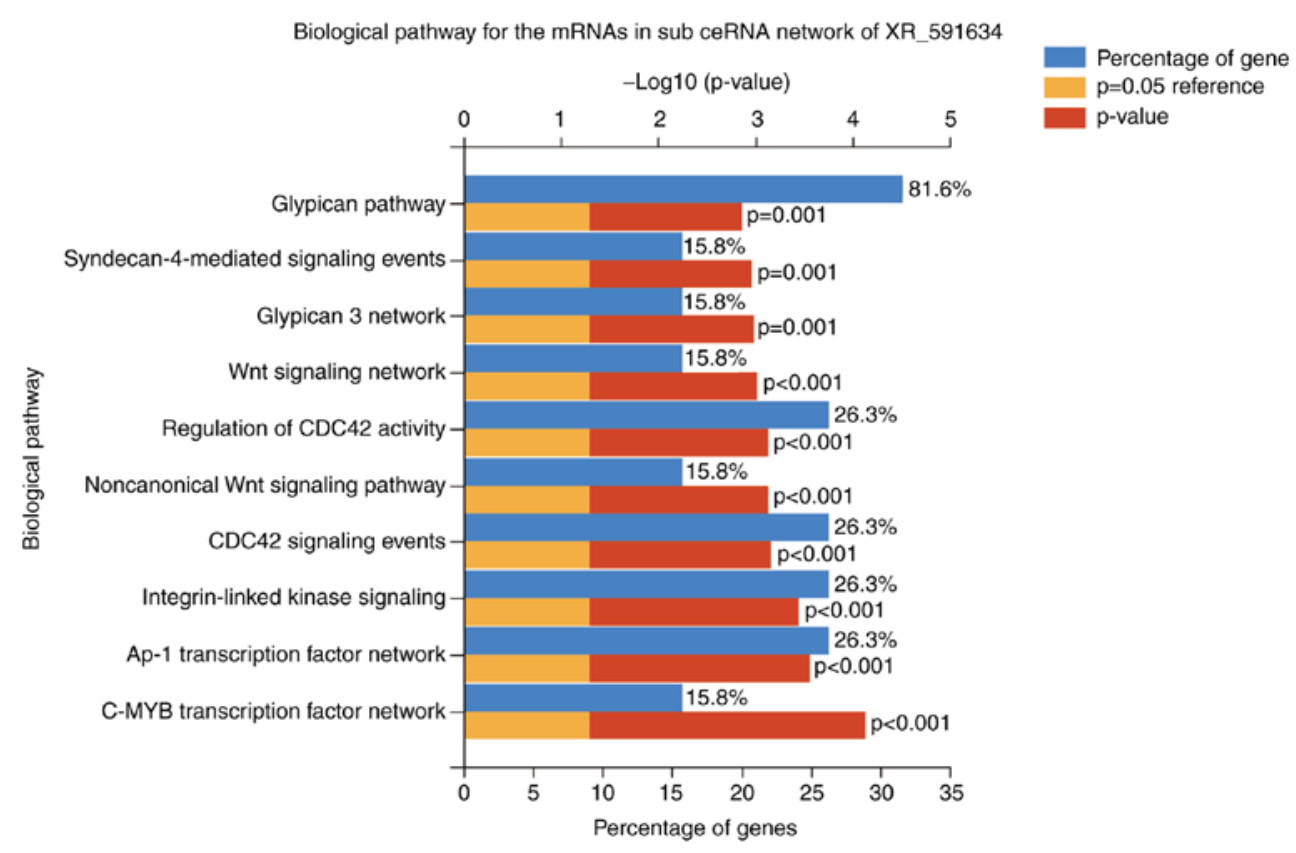

Figure 8. Top 10 biological pathways of the mRNAs in the sub-ceRNA network of the long noncoding RNA XR_591634. The pathways included the C-MYB transcription factor network, AP-1 transcription factor network, integrin-linked kinase signaling, CDC42 signaling events, noncanonical Wnt signaling pathway, regulation of CDC42 activity, Wnt signaling network, glypican 3 network, syndecan-4-mediated signaling events and glypican pathway. mRNA, messenger RNA; ceRNA, competing endogenous RNA; AP-1, activator protein 1; CDC42, cell division cycle 42.

CDC42 activity, Wnt signaling network, glypican 3 network, syndecan-4-mediated signaling events, and Glypican pathway (Fig. 8).

Comparing the results of these three subnetworks, XR_350851 was observed to be associated with the classic autophagy pathway, namely the $\mathrm{PI} 3 \mathrm{~K} / \mathrm{Akt} / \mathrm{mTOR}$ signaling pathway. The interactions of the three lncRNA-targeted mRNAs were also enriched using FunRich software (Figs. 9-11).

\section{Discussion}

Approximately 130,000 people, predominantly young adults, suffer from SCI paralysis annually worldwide, which has 


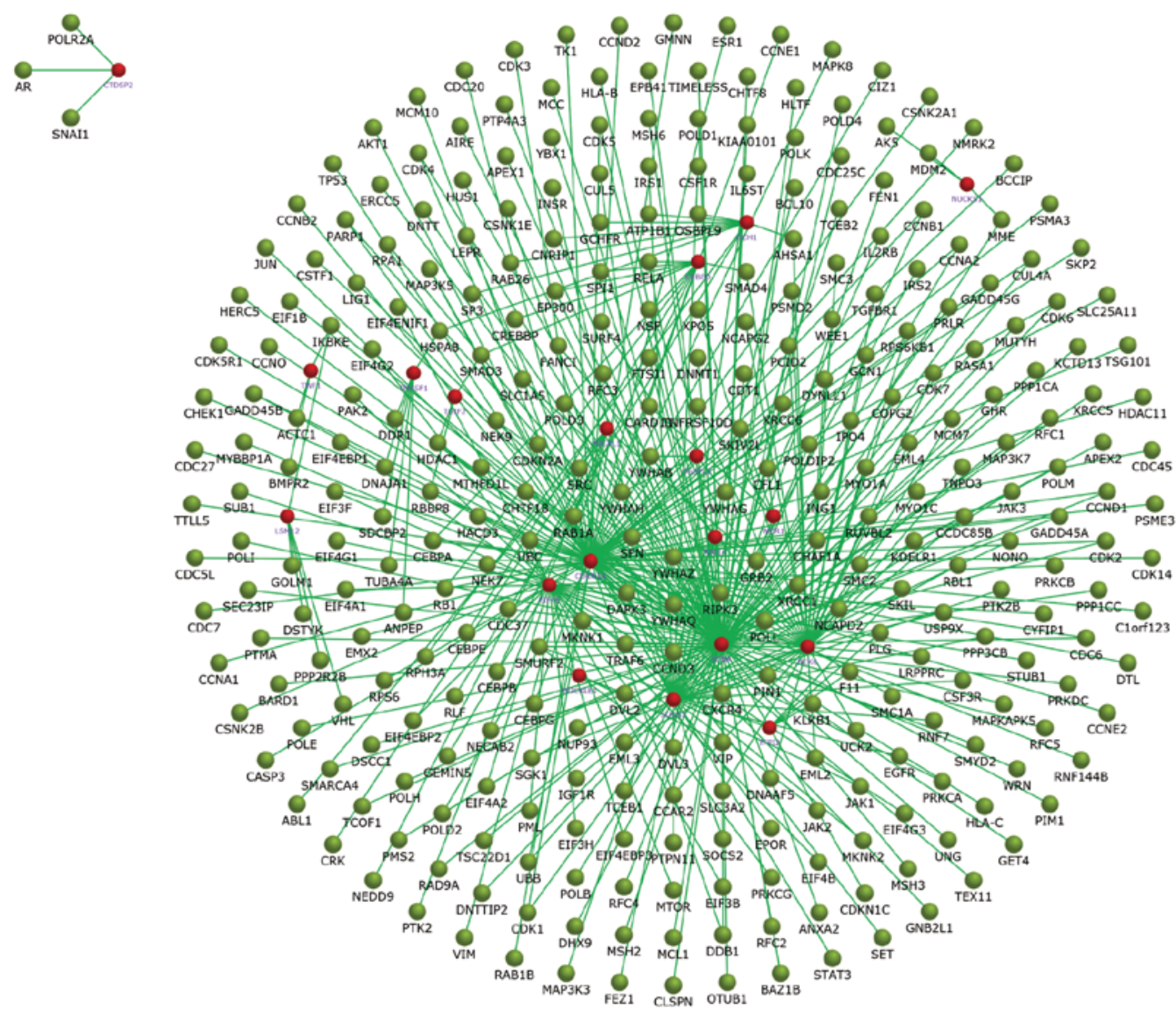<smiles>O=S(=O)(O)OC1CCCCC1</smiles>
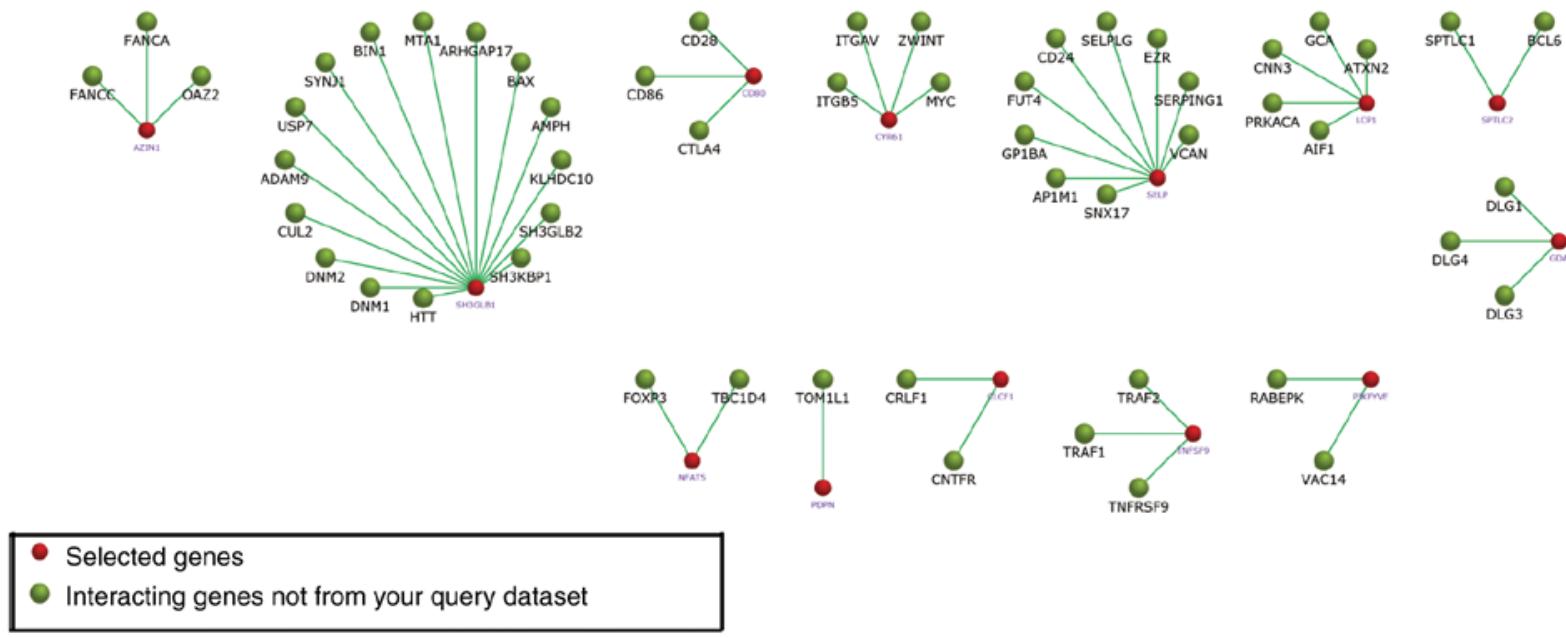

Interacting genes not from your query dataset

Figure 9. Protein-protein interaction network of XR_350851-targeted messenger RNAs. Red circles indicate the selected genes, while green circles indicatenodes from outside the selected dataset that exhibited interactions.

a serious socioeconomic impact (37). The complex pathophysiology of SCI, which includes primary and secondary mechanisms, involves a complicated cascade of molecules and hampers the generation of effective therapies (38). To identify target lncRNAs that have potential as novel predictors in clinical diagnoses and treatments, a network based on the ceRNA theory was generated by mining lncRNA, miRNA and mRNA data from NCBI GEO database. The resulting lncRNA-miRNA-mRNA regulatory network consisted of 13 lncRNA, 93 mRNA and 9 miRNA nodes, with a total of 202 edges.

Molecular function and biological pathway analyses were used to assign the biological functions of differentially expressed genes. The module analysis identified 75 molecular function modules associated with SCI. The identified molecular functions included calcium ion-binding, cysteine-type peptidase activity, chemokine activity, auxiliary transport protein activity, cytokine activity, hormone binding, steroid binding, protein-tyrosine kinase activity, transcription factor activity and cytoskeletal anchoring activity. In the current study, it was observed that the node degrees of three lncRNAs, namely XR_350851, NR_027820 and XR_591634, were significantly higher compared with those of other lncRNAs. These three lncRNAs also exhibited an increased number of lncRNA-miRNA and miRNA-mRNA pairs. Therefore, it is 

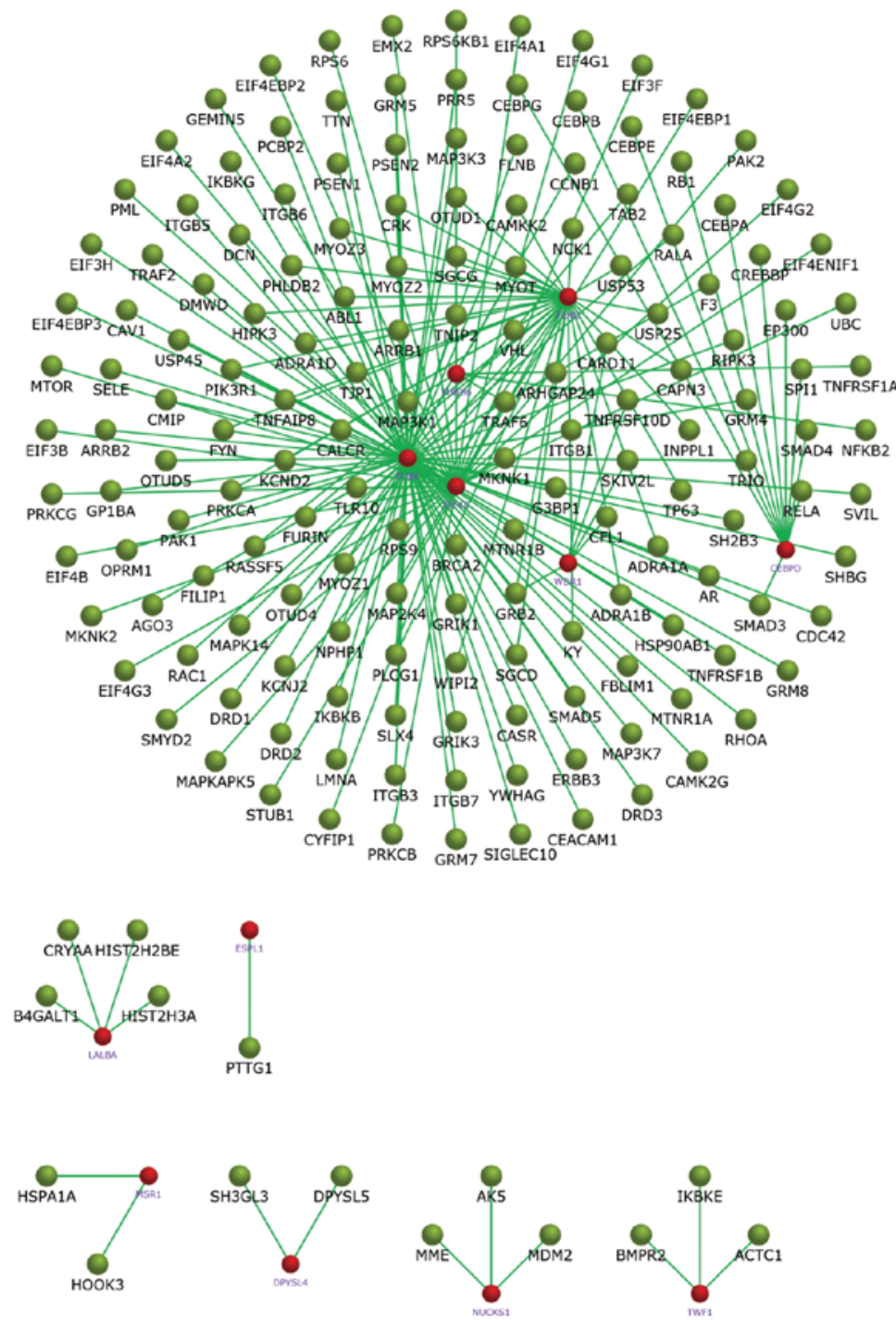

- Selected genes

Interacting genes not from your query dataset

Figure 10. Protein-protein interaction network of NR_027820-targeted messenger RNAs. Red circles indicate the selected genes, while green circles indicate nodes from outside the selected dataset that exhibited interactions.

suggested that these lncRNAs (XR_350851, NR_027820 and XR_591634) may have profound implications in SCI andmay be potential target lncRNAs. Subsequently, sub-ceRNA networks of the three aforementioned node lncRNAs were reconstructed in the current study.

Biological pathway analysis thenidentified that 263, 139 and 174 pathways were respectively enriched in the XR_350851, NR_027820 and XR_591634 subnetworks. The predominant biological pathways in the XR_350851 subnetwork were the AP-1 transcription factor network, S1P1 pathway, class I PI3K signaling events mediated by Akt, insulin pathway and mTOR signaling pathway. The significant biological pathways in the NR_027820 subnetwork included platelet degranulation, cell junction organization, ceramide biosynthesis, hemostasis and platelet activation. In addition, the AP-1 transcription factor network, integrin-linked kinase signaling, regulation of CDC42 activity, Wnt signaling network and glypican pathway were the critical biological pathways in the XR_591634 subnetwork.
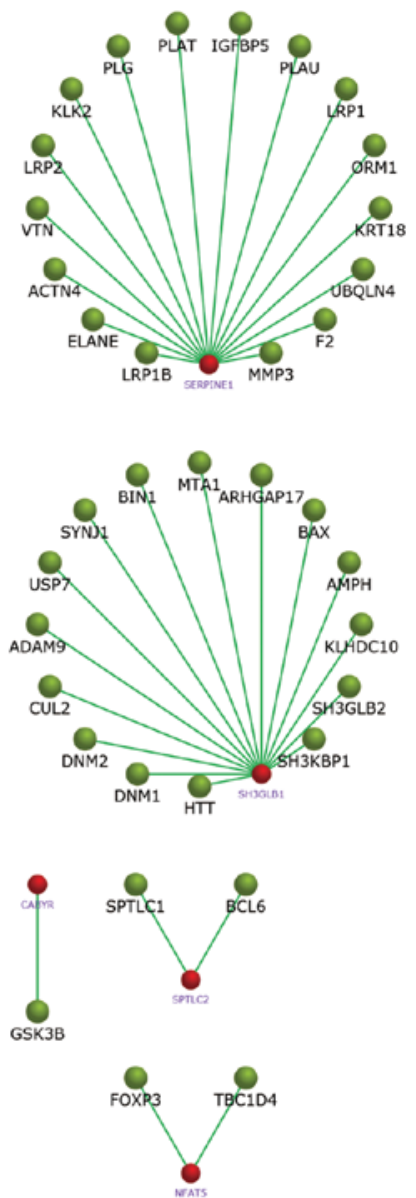

A previous study by Tsuboyama et al (39) determined that starvation conditions or mTOR inhibition promoted VPS34-dependent ribophagic flux in cells. In addition, a previous study reported that rapamycin may aid in the restoration of motor function and act in a neuroprotective manner by suppressing the mTOR pathway (40). As a central regulator of autophagy and a serine/threonine kinase, mTOR is involved in cancer, andin cardiovascular and neurological diseases. In addition, mTOR is the catalytic subunit of two distinct signaling complexes, including mTOR complex 1 (mTORC1) and mTORC2 $(41,42)$, which are significantly involved in the control of cell proliferation. It is noteworthy that the growth factor/PI3K/Akt signaling pathway is the upstream modulator of mTORC1 (43). Another important finding was that mTORC2-ribosome binding can be improved by insulin stimulation of the PI3K signaling pathway (44). There is increasing evidence that inhibition of the autophagy-associated mTOR pathway subsequent to SCI may have a neuroprotective effect (39). Furthermore, Bai et al (45) revealed that stimulating 

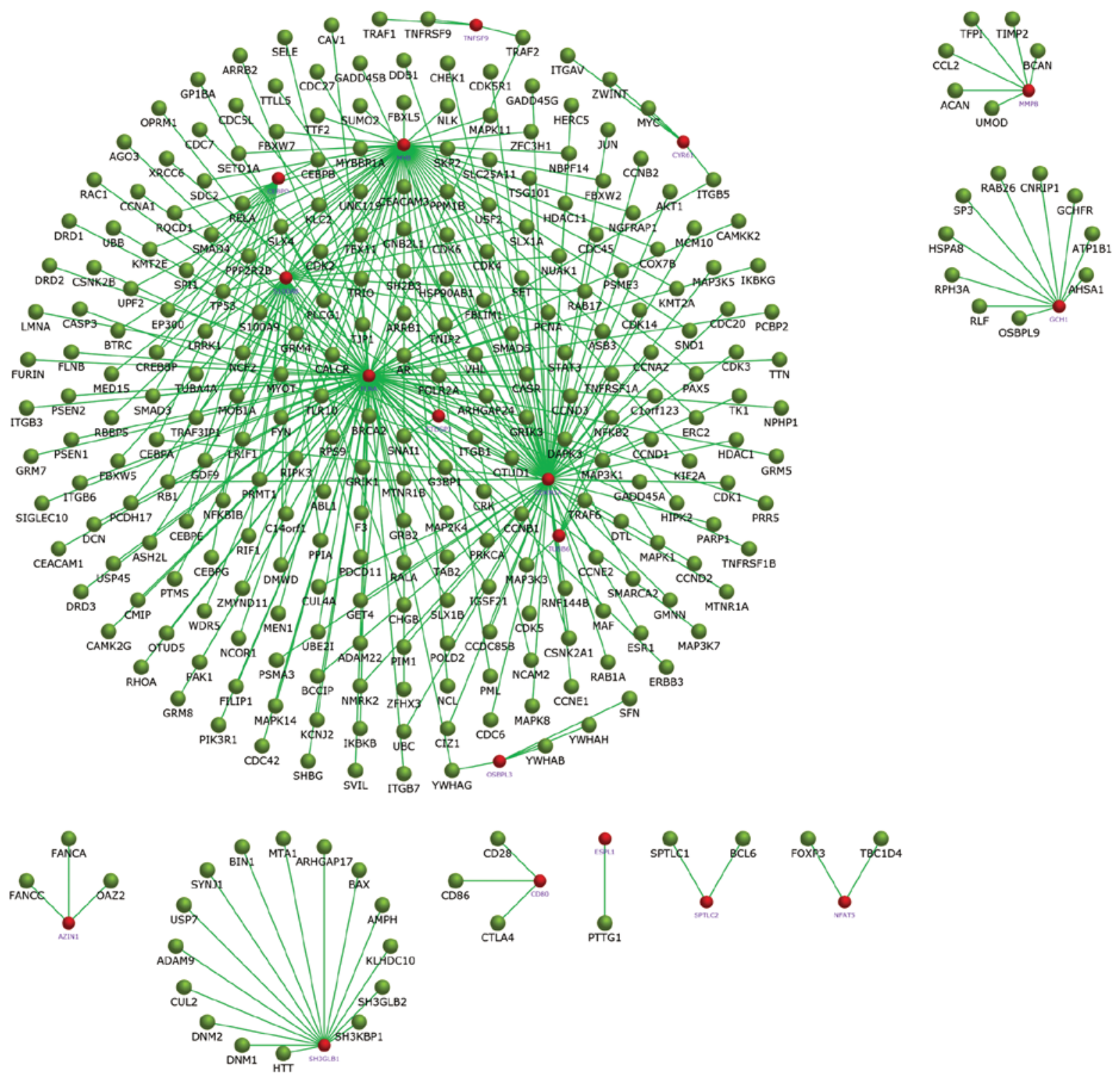

Interacting genes not from your query dataset

Figure 11. Protein-protein interaction network of XR_591634-targeted messenger RNAs. Red circles indicate the selected genes, while green circles indicate nodes from outside the selected dataset that exhibited interactions.

the AMPK/mTOR signaling pathway of autophagy may facilitate functional recovery from SCI. The PI3K/Akt/mTOR pathway has previously been identified as a classic autophagy pathway (46). In addition, the PI3K/Akt/mTOR pathway is important for XR_350851 associated mRNA. Therefore, XR_350851 may be used as a target gene for neuroprotection following SCI. However, whether the IncRNA XR_350851 regulates autophagy in SCI has yet to be determined. Therefore, it is suggested that future studies should focus on the role of XR_350851 in SCI.

In conclusion, using mined data and based on the ceRNA theory, an SCI-associated lncRNA-miRNA-mRNA network was constructed in the current study. According to the distribution of the nodes in the ceRNA network, three lncRNAs (XR_350851, NR_027820 and XR_591634) were identified, and their sub-ceRNA networks constructed. Furthermore, the functions of these three node IncRNAs were predicted by enriching the pathways of their associated mRNAs in the sub-ceRNA networks. The present study provided further insight into the involvement of lncRNAs in the mechanism of SCI, and the identified lncRNAs may serve as potential novel biomarkers and therapeutic targets for SCI. Finally, it is speculated that the lncRNA XR_350851 is closely associated with autophagy. However, further research is required to determine the biological role of XR_350851 in SCI.

\section{Acknowledgements}

Not applicable.

\section{Funding}

No funding was received. 


\section{Availability of data and materials}

The datasets used and/or analyzed during the current study are available from the corresponding author on reasonable request.

\section{Authors' contributions}

ZQ conceived and designed the present study. LW, JL and BW collected, extracted and analyzed the data. LW wrote the manuscript. LW and ZQ reviewed the final manuscript. All authors read and approved the final manuscript.

\section{Ethics approval and consent to participate}

Not applicable.

\section{Patient consent for publication}

Not applicable.

\section{Competing interests}

The authors declare that they have no competing interests.

\section{References}

1. Ramer LM, Ramer MS and Bradbury EJ: Restoring function after spinal cord injury: Towards clinical translation of experimental strategies. Lancet Neurol 13: 1241-1256, 2014.

2. Su M, Guan H, Zhang F, Gao Y, Teng X and Yang W: HDAC6 regulates the chaperone-mediated autophagy to prevent oxidative damage in injured neurons after experimental spinal cord injury. Oxid Med Cell Longev 2016: 7263736, 2016.

3. Walker CL, Walker MJ, Liu NK, Risberg EC, Gao X, Chen J and Xu XM: Systemic bisperoxovanadium activates Akt/mTOR reduces autophagy and enhances recovery following cervical spinal cord injury. PLoS One 7: e30012, 2012.

4. Thuret S, Moon LD and Gage FH: Therapeutic interventions after spinal cord injury. Nat Rev Neurosci 7: 628-643, 2006.

5. Li GL, Brodin G, Farooque M, Funa K, Holtz A, Wang WL and Olsson Y: Apoptosis and expression of Bcl-2 after compression trauma to rat spinal cord. J Neuropathol Exp Neurol 55: 280-289, 1996.

6. Galluzzi L, Bravo-San Pedro JM, Blomgren K and Kroemer G: Autophagy in acute brain injury. Nat Rev Neurosci 17: 467-484, 2016.

7. Ohsumi Y: Historical landmarks of autophagy research. Cell Res 24: 9-23, 2014.

8. Chen ZH, Wang WT, Huang W, Fang K, Sun YM, Liu SR, Luo XQ and Chen YQ: The IncRNA HOTAIRM1 regulates the degradation of PML-RARA oncoprotein and myeloid cel differentiation by enhancing the autophagy pathway. Cell Death Differ 24: 212-224, 2017.

9. Kroemer G: Autophagy: A druggable process that is deregulated in aging and human disease. J Clin Invest 125: 1-4, 2015.

10. Yang L, Wang H, Shen Q, Feng L and Jin H: Long non-coding RNAs involved in autophagy regulation. Cell Death Dis 8: e3073, 2017.

11. Pott J, Kabat AM and Maloy KJ: Intestinal epithelial cell autophagy is required to protect against TNF-induced apoptosis during chronic colitis in mice. Cell Host Microbe 23: 191-202. e4, 2018.

12. Papadakis M, Hadley G, Xilouri M, Hoyte LC, Nagel S, McMenamin MM, Tsaknakis G, Watt SM, Drakesmith CW, Chen R, et al: Tsc1 (hamartin) confers neuroprotection against ischemia by inducing autophagy. Nat Med 19: 351-357, 2013.

13. Wu F, Wei X, Wu Y, Kong X, Hu A, Tong S, Liu Y, Gong F, Xie L, Zhang J, et al: Chloroquine promotes the recovery of acute spinal cord injury by inhibiting autophagy-associated inflammation and endoplasmic reticulum stress. J Neurotrauma 35: 1329-1344, 2018.
14. Fritah S, Niclou SP and Azuaje F: Databases for lncRNAs: A comparative evaluation of emerging tools. RNA 20: 1655-1665, 2014.

15. Gu S, Xie R, Liu X, Shou J, Gu W and Che X: Long coding RNA XIST contributes to neuronal apoptosis through the downregulation of AKT phosphorylation and is negatively regulated by miR-494 in rat spinal cord injury. Int J Mol Sci 18: pii: E732, 2017.

16. Qiao Y, Peng C, Li J, Wu D and Wang X: LncRNA MALAT1 is neuroprotective in a rat model of spinal cord ischemia-reperfusion injury through miR-204 regulation. Curr Neurovasc Res 15: 211-219, 2018.

17. Zhou HJ, Wang LQ, Wang DB, Yu JB, Zhu Y, Xu QS, Zheng XJ and Zhan RY: Long noncoding RNA MALAT1 contributes to inflammatory response of microglia following spinal cord injury via the modulation of a miR-199b/IKK $\beta / \mathrm{NF}-\kappa \mathrm{B}$ signaling pathway. Am J Physiol Cell Physiol 315: C52-C61, 2018.

18. Tay Y, Rinn J and Pandolfi PP: The multilayered complexity of ceRNA crosstalk and competition. Nature 505: 344-352, 2014.

19. Salmena L, Poliseno L, Tay Y, Kats L and Pandolfi PP: A ceRNA hypothesis: The Rosetta Stone of a hidden RNA language? Cell 146: 353-358, 2011

20. Xu J, Li Y, Lu J, Pan T, Ding N, Wang Z, Shao T, Zhang J, Wang $\mathrm{L}$ and Li X: The mRNA related ceRNA-ceRNA landscape and significance across 20 major cancer types. Nucleic Acids Res 43: 8169-8182, 2015.

21. Zhang X, Sun S, Pu JK, Tsang AC, Lee D, Man VO, Lui WM, Wong ST and Leung GK: Long non-coding RNA expression profiles predict clinical phenotypes in glioma. Neurobiol Dis 48: $1-8,2012$.

22. Johnson WE, Li C and Rabinovic A: Adjusting batch effects in microarray expression data using empirical Bayes methods. Biostatistics 8: 118-127, 2007.

23. Leek JT, Scharpf RB, Bravo HC, Simcha D, Langmead B, Johnson WE, Geman D, Baggerly $K$ and Irizarry RA: Tackling the widespread and critical impact of batch effects in high-throughput data. Nat Rev Genet 11: 733-739, 2010.

24. Leek JT, Johnson WE, Parker HS, Jaffe AE and Storey JD: The sva package for removing batch effects and other unwanted variation in high-throughput experiments. Bioinformatics 28: 882-883, 2012.

25. Yang X, Zhu S, Li L, Zhang L, Xian S, Wang Y and Cheng Y: Identification of differentially expressed genes and signaling pathways in ovarian cancer by integrated bioinformatics analysis. Onco Targets Ther 11: 1457-1474, 2018.

26. Rehmsmeier M, Steffen P, Hochsmann M and Giegerich R: Fast and effective prediction of microRNA/target duplexes. RNA 10: 1507-1517, 2004

27. Li J, Ma W, Zeng P, Wang J, Geng B, Yang J and Cui Q: LncTar: A tool for predicting the RNA targets of long noncoding RNAs. Brief Bioinform 16: 806-812, 2015.

28. Agarwal V, Bell GW, Nam JW and Bartel DP: Predicting effective microRNA target sites in mammalian mRNAs. Elife 4, 2015.

29. Paraskevopoulou MD, Georgakilas G, Kostoulas N, Vlachos IS, Vergoulis T, Reczko M, Filippidis C, Dalamagas T and Hatzigeorgiou AG: DIANA-microT web server v5.0: Service integration into miRNA functional analysis workflows. Nucleic Acids Res 41: (Web Server Issue) W169-W173, 2013.

30. Reczko M, Maragkakis M, Alexiou P, Grosse I and Hatzigeorgiou AG: Functional microRNA targets in protein coding sequences. Bioinformatics 28: 771-776, 2012.

31. Lewis BP, Burge CB and Bartel DP: Conserved seed pairing, often flanked by adenosines, indicates that thousands of human genes are microRNA targets. Cell 120: 15-20, 2005.

32. Jiang H, Ma R, Zou S, Wang Y, Li Z and Li W: Reconstruction and analysis of the IncRNA-miRNA-mRNA network based on competitive endogenous RNA reveal functional lncRNAs in rheumatoid arthritis. Mol Biosyst 13: 1182-1192, 2017.

33. Sun J, Yan J, Yuan X, Yang R, Dan T, Wang X, Kong G and Gao S: A computationally constructed ceRNA interaction network based on a comparison of the SHEE and SHEEC cell lines. Cell Mol Biol Lett 21: 21, 2016.

34. Morris JH, Wu A, Yamashita RA, Marchler-Bauer A and Ferrin TE: cddApp: A Cytoscape app for accessing the NCBI conserved domain database. Bioinformatics 31: 134-136, 2015.

35. Pathan M, Keerthikumar S, Ang CS, Gangoda L, Quek CY, Williamson NA, Mouradov D, Sieber OM, Simpson RJ, Salim A, et al: FunRich: An open access standalone functional enrichment and interaction network analysis tool. Proteomics 15: 2597-2601, 2015. 
36. Zhou M, Diao Z, Yue X, Chen Y, Zhao H, Cheng L and Sun J: Construction and analysis of dysregulated lncRNA-associated ceRNA network identified novel lncRNA biomarkers for early diagnosis of human pancreatic cancer. Oncotarget 7: 56383-56394, 2016.

37. Jackson A and Zimmermann JB: Neural interfaces for the brain and spinal cord-restoring motor function. Nat Rev Neurol 8: 690-699, 2012.

38. Blesch A and Tuszynski MH: Spinal cord injury: Plasticity, regeneration and the challenge of translational drug development. Trends Neurosci 32: 41-47, 2009.

39. Tsuboyama K, Koyama-Honda I, Sakamaki Y, Koike M, Morishita $\mathrm{H}$ and Mizushima N: The ATG conjugation systems are important for degradation of the inner autophagosomal membrane. Science 354: 1036-1041, 2016.

40. Sekiguchi A, Kanno H, Ozawa H, Yamaya S and Itoi E: Rapamycin promotes autophagy and reduces neural tissue damage and locomotor impairment after spinal cord injury in mice. J Neurotrauma 29: 946-956, 2012.

41. Ge D, Han L, Huang S, Peng N, Wang P, Jiang Z, Zhao J, Su L, Zhang S, Zhang Y, et al: Identification of a novel MTOR activator and discovery of a competing endogenous RNA regulating autophagy in vascular endothelial cells. Autophagy 10: 957-971, 2014.
42. Chen JF, Wu P, Xia R, Yang J, Huo XY, Gu DY, Tang CJ, De W and Yang F: STAT3-induced lncRNA HAGLROS overexpression contributes to the malignant progression of gastric cancer cells via mTOR signal-mediated inhibition of autophagy. Mol Cancer 17: 6, 2018.

43. Kim YC and Guan KL: mTOR: A pharmacologic target for autophagy regulation. J Clin Invest 125: 25-32, 2015.

44. Zinzalla V, Stracka D, Oppliger W and Hall MN: Activation of mTORC2 by association with the ribosome. Cell 144: 757-768, 2011.

45. Bai L, Mei X, Shen Z, Bi Y, Yuan Y, Guo Z, Wang H, Zhao H, Zhou Z, Wang C, et al: Netrin-1 improves functional recovery through autophagy regulation by activating the AMPK/mTOR signaling pathway in rats with spinal cord injury. Sci Rep 7: 42288, 2017.

46. Saiki S, Sasazawa Y, Imamichi Y, Kawajiri S, Fujimaki T, Tanida I, Kobayashi H, Sato F, Sato S, Ishikawa K, et al: Caffeine induces apoptosis by enhancement of autophagy via PI3K/Akt/mTOR/p70S6K inhibition. Autophagy 7: 176-187, 2011.

This work is licensed under a Creative Commons Attribution-NonCommercial-NoDerivatives 4.0 International (CC BY-NC-ND 4.0) License. 